\title{
Journal of Clinical Immunology
}

\section{Methicillin-Resistant Staphylococcus Aureus (MRSA): Prevalence and Antimicrobial Sensitivity Pattern among Outdoor and Indoor Patients Dhaka City Hospital, Bangladesh}

\author{
Md. Ashiqur Rahman ${ }^{\text {* }}$ \\ ${ }^{1}$ M.Sc and B.Sc, Department of Microbiology, Stamford University, Bangladesh \\ *Corresponding Author: Md. Ashiqur Rahman, M.Sc and B.Sc, Department of Microbiology, Stamford \\ University, Bangladesh; Email: ashiqmbo@gmail.com
}

Received Date: 03-11-2020; Accepted Date: 25-11-2020; Published Date: 04-12-2020

Copyright $^{\oplus} 2020$ by Rahman AM. All rights reserved. This is an open access article distributed under the terms of the Creative Commons Attribution License, which permits unrestricted use, distribution, and reproduction in any medium, provided the original author and source are credited.

\begin{abstract}
Methicillin-Resistant Staphylococcus Aureus (MRSA) is one of the significant pathogens with associated public-health concern in the world. This present study has been focused on the prevalence of MRSA and their antibiotic resistance of indoor and outdoor patients different age and gender in clinical history, Dr. Sirajul Islam medical college and hospital Dhaka, Bangladesh. We were performed during the period of January 2019 to July 2019. A total 964 samples which is outdoor 354 (36.72\%) indoor 610 (63.27\%). We were observation 5 days (BACTEC-9050 Machine) then inoculated selective onto Blood agar and MacConkey agar media and the culture plates were incubated at $37^{\circ} \mathrm{C}$ for $24 \mathrm{hrs}$. The $S$. aureus colonies showing golden yellow colour on Blood agar were primarily identified as $S$. aureus, which were then subjected for catalase and coagulase tests. The Methicillin-Resistant Staphylococcus Aureus (MRSA) could be detected only $42(4.35 \%)$ into, males $18(1.86 \%)$ and female $24(2.48 \%)$ in total sample, on the other hand Staphylococci aureus. (MSSA) 50 (5.18\%), Pseudomonas spp. 14 (1.45\%), Klebsiella spp. 13 (1.34\%), Salmonella spp. 27 (2.80\%), E. coli 21 (2.17\%), No MRSA, MSSA and other organisms are found 797 (82.67\%), Standard antimicrobial disks representing multiple sixteen drug were, Sensitive-381 (56.69\%), Resistance-284 (42.26\%), as well as Intermediate-7 (1.04\%) against antibiotics, Oxacillin, Vancomycin, Erythromycin, Cotrimoxazole, Ciprofloxacin, Gentamicin, Linezolid, Amoxicillin, Amoxyclave, Azithromycin,
\end{abstract}


Imipenem, Meropenem, Netilmicin, Gatifloxacin, Cloxacillin, Sparfloxacin. In this review, we describe the current burden of MRSA infections in healthcare and community of Bangladesh. And settings across other country the main threats caused by recent changes in the epidemiology of MRSA. This data has important implication for quality of patients care antibiotic selection and infection control practices.

\section{Keywords}

Methicillin-Resistant Staphylococcus Aureus; Hospital; Indoor Outdoor Patients; Blood; Antimicrobial Sensitivity

\section{Introduction}

In today's world Antimicrobial Resistance (AMR) is a vital public-health concern. It is true that Methicillin-Resistant Staphylococcus Aureus (MRSA) is one of the significant pathogens in worldwide as well as is associated with high morbidity and mortality amount with rapid development of resistance [1,2]. Science 1960s, S. aureus initially described, on the other hand, S. aureus, is principally amalgamated with skin, skin glands and mucous membrane of warmblooded animals so an adaptable microbe as well as a frequent invader of animals, and humans [3]. The organism is favour nosocomial bacteria implicate with several infections, including pneumonia, septic arthritis, osteomyelitis, endocarditis, toxic-shock syndrome and food poisoning [4-7]. Many scientists are of the opinion that, (healthcare associated methicillinresistant $S$. aureus, HA-MRSA), the burden of MRSA colonisation and infection has recently enlarged to further ecological slot [8]. After that 1990s, an increasing prevalence of MRSA infections emerge in the community (Community Associated Methicillin-Resistant Staphylococcus Aureus, CA-MRSA), has been described from many countries globally $[9,10]$. Research show that, Bangladesh is a high prevalence of MRSA infection, where the proportion is greater than three quarter has been observed in Asian several countries, including Japan, India, Pakistan, Thailand and Vietnam [11]. Those countries as well experience a high incidence of MRSA infection [12,13]. In the present study, we estimated the prevalence of $S$. aureus and MRSA in blood sample indoor as well as outdoor medical patient whereas antimicrobial resistance typing was used to determine the relatedness among the MRSA isolates.

\section{Material and Method}

\section{Sample Collection}

A total of 964 blood sample were cultured for the isolation of $S$. aureus from patients in hospitals, (out door and in door) Dr Sirajul Islam medical college and hospital Ltd. S. aureus 
was identified and antibiotic susceptibility tests were performed in the Department of Microbiology. The tests were performed during the period of January 2019 to July 2019. Records including clinical history, type of infection, gender, age, consumption of antibiotics, were obtained from patients whose sample S. aureus (MRSA) had been isolated from Blood agar and MacConkey agar media [14].

\section{Isolation and Identification of S. aureus}

Isolation and enumeration of staphylococci was carried out as previously described. Enumeration was performed only in blood sample. At first, we were collected blood sample from collection department, then we were preserving blood sample 5 days (BACT 9050 machine) while as is observed machine called positive signal, we were sub culture selective on Blood agar and MacConkey agar media. Then microorganism's primary identified by aerobically incubated for 24 hours at $37^{\circ} \mathrm{C}$ golden yellow colony. Secondary recognized by characterization on blood agar were subjected to gram staining, catalase test, and coagulase test [15].

\section{Antimicrobial Sensitivity Testing}

Antimicrobial susceptibility of the $S$. aureus against 16 antimicrobials was determined by the agar-dilution method in Mueller-Hinton agar. According to the Clinical and Laboratory Standard Institute (CLSI) guidelines and the results were appraised after incubation at $37^{\circ} \mathrm{C}$ for 24 hours [16]. The final plate combination $(\mathrm{mg} / \mathrm{mL}$ ) were used (Oxoid, UK) antibiotics, Oxacillin, Vancomycin, Erythromycin, Co-trimoxazole, Ciprofloxacin, Gentamicin, Linezolid, Amoxicillin, Amoxyclave, Azithromycin, Imipenem, Meropenem, Netilmicin, Gatifloxacin, Cloxacillin, Sparfloxacin.

\section{Results and Discussion}

\section{Isolation of Methicillin Resistant Staphylococcus Aureus}

Methicillin-Resistant Staphylococcus Aureus (MRSA) (green yellowish colony) was isolated from $42(4.35 \%)$ out of the 964 blood samples analysed. The isolation frequencies outdoor and indoor patient Dr Sirajul Islam Medical College and Hospital Ltd. Sample type is shown in (Table 1). January was the first month we were collected 86 blood sample in which present 3 $(0.31 \%)$ patients MRSA, and February 79 sample were present $1(0.10 \%)$ patients MRSA in indoor patients. On the other hand, the number of MRSA is present indoor \& outdoor patients is $5(0.51 \%)$ and $1(0.10 \%)$ in March out of 112 sample. Between April and May, the number

Rahman AM | Volume 1; Issue 2 (2020) | JCIM-1(2)-010 | Research Article

Citation: Rahman AM. Methicillin-Resistant Staphylococcus Aureus (MRSA): Prevalence and Antimicrobial Sensitivity Pattern among Outdoor and Indoor Patients Dhaka City Hospital, Bangladesh. J Clin Immunol Microbiol. 2020;1(2):1-9. 
of MRSA blood sample was same indoor patients $4(0.41 \%), 4(0.41 \%)$ as well as outdoor patients in April was $1(0.10 \%)$ and May is $0(0.00 \%)$ from 103 and 117 blood sample. The number of MRSA abruptly increased from indoor patients $10(1.03 \%)$ and $11(1.14 \%)$ outdoor patients $2(0.20 \%), 0(0.00 \%)$ between June and July in Sirajul Islam Medical College and Hospital Ltd.

\begin{tabular}{|l|l|l|l|l|l||}
\hline \multirow{2}{*}{ Period } & \multirow{2}{*}{ Total Blood Sample } & \multicolumn{2}{|l|}{ Identify MRSA Indoor Patients } & \multicolumn{2}{l|}{ Identify MRSA Out-door Patients } \\
\cline { 3 - 6 } & & Present & Absent & Present & Absent \\
\hline January & 86 & $3(0.31 \%)$ & $53(5.49 \%)$ & $0(0.00 \%)$ & $30(3.11 \%)$ \\
\hline February & 79 & $1(0.10 \%)$ & $38(3.94 \%)$ & $0(0.00 \%)$ & $40(4.14 \%)$ \\
\hline March & 112 & $5(0.51 \%)$ & $65(6.74 \%)$ & $1(0.10 \%)$ & $41(4.25 \%)$ \\
\hline April & 103 & $4(0.41 \%)$ & $79(8.19 \%)$ & $1(0.10 \%)$ & $19(1.97 \%)$ \\
\hline May & 117 & $4(0.41 \%)$ & $83(8.60 \%)$ & $0(0.00 \%)$ & $30(3.11 \%)$ \\
\hline June & 131 & $10(1.03 \%)$ & $65(6.74 \%)$ & $2(0.20 \%)$ & $54(5.60 \%)$ \\
\hline July & 336 & $11(1.14 \%)$ & $189(19.60 \%)$ & $0(0.00 \%)$ & $136(14.10 \%)$ \\
\hline Total & $\mathbf{9 6 4}$ & $\mathbf{3 8 ( 3 . 9 4 \% )}$ & $\mathbf{5 7 2}(\mathbf{5 9 . 3 3 \% )}$ & $\mathbf{4 ( 0 . 4 1 \% )}$ & $\mathbf{3 5 0}(\mathbf{3 6 . 3 0 \% )}$ \\
\hline
\end{tabular}

Table 1: Prevalence of methicillin-resistant Staphylococcus aureus isolates and classified by period of collection, indoor \& outdoor patients. Dr Sirajul Islam medical college and hospital Ltd.

\section{Antibiotic Susceptibility of MRSA Isolates:}

According to antibiogram outline, the 42 (4.35\%) MRSA isolates from 964 (100\%) indoor and outdoor patients blood sample. The fact of the matter is, Cefoxitin disk dispersion test we were used to screen all $S$. aureus isolates. Isolates with a zone of growth inhibition $\geq 22 \mathrm{~mm}$ are defined as methicillin-sensitive $S$. aureus (MSSA), and those with a zone diameter $<21 \mathrm{~mm}$ as Methicillin Resistant S. Aureus (MRSA) [16]. Where resistant and sensitive against Oxacillin $\mathrm{S}=4$ (0.59\%), $\mathrm{R}=38$ (5.65\%), Ciprofloxacin $\mathrm{S}=33$ (4.91\%) $\mathrm{R}=8$ (1.19\%) $\mathrm{I}=1$ (0.14\%), Gentamicin $\mathrm{S}=22$ (3.27\%) $\mathrm{R}=20$ (2.97\%), Amoxicillin $\mathrm{R}=30$ (4.46\%) $\mathrm{S}=12$ (1.78\%), Cloxacillin $\mathrm{S}=5$ (0.74\%) $\mathrm{R}=37$ (5.50\%), Sparfloxacin $\mathrm{S}=24$ (3.57\%) $\mathrm{R}=18$ (2.67\%), Vancomycin $\mathrm{S}=40$ (5.95\%) R=2 (0.29\%), Erythromycin R=10 (1.48\%) $\mathrm{S}=30$ (4.46\%) $\mathrm{I}=2$ (0.29\%), Co-trimoxazole $\mathrm{R}=17$ (2.52\%) $\mathrm{S}=25$ (3.72\%), Linezolid $\mathrm{S}=36(5.35 \%) \mathrm{R}=6$ (0.89\%), 
Amoxyclave $\mathrm{R}=14$ (2.08\%) $\mathrm{S}=28$ (4.16\%), Azithromycin I=3 (0.44\%) $\mathrm{S}=22$ (3.27\%) $\mathrm{R}=17$ (2.52\%), Imipenem $\mathrm{R}=9$ (1.33\%) $\mathrm{S}=33$ (4.91\%), Meropenem $\mathrm{S}=21$ (3.12\%) $\mathrm{R}=21$ (3.12\%), Netilmicin $I=1(0.29 \%) \mathrm{R}=4$ (0.59\%) $\mathrm{S}=37$ (5.50\%), Gatifloxacin $\mathrm{S}=34(5.05 \%) \mathrm{R}=8(1.19 \%)$. ( $\mathrm{R}=$ Resistance, $\mathrm{S}=$ Sensitive, $\mathrm{I}=$ Intermediate) (Table 2).

\begin{tabular}{|c|c|c|c|}
\hline \multirow[t]{2}{*}{ Antibiotic } & \multicolumn{3}{|c|}{ Indoor and Outdoor Patients (MRSA) 42 (4.35\%) } \\
\hline & Sensitive & Resistant & Intermediate \\
\hline Oxacillin & $4(0.59 \%)$ & $38(5.65 \%)$ & \\
\hline Vancomycin & $40(5.95 \%)$ & $2(0.29 \%)$ & \\
\hline Erythromycin & $30(4.46 \%)$ & $10(1.48 \%)$ & $2(0.29 \%)$ \\
\hline Co-trimoxazole & $25(3.72 \%)$ & $17(2.52 \%)$ & \\
\hline Ciprofloxacin & $8(1.19 \%)$ & $33(4.91 \%)$ & $1(0.14 \%)$ \\
\hline Gentamicin & $22(3.27 \%)$ & $20(2.97 \%)$ & \\
\hline Linezolid & $36(5.35 \%)$ & $6(0.89 \%)$ & \\
\hline Amoxicillin & $12(1.78 \%)$ & $30(4.46 \%)$ & \\
\hline Amoxyclave & $28(4.16 \%)$ & $14(2.08 \%)$ & \\
\hline Azithromycin & $22(3.27 \%)$ & $17(2.52 \%)$ & $3(0.44 \%)$ \\
\hline Imipenem & $33(4.91 \%)$ & $9(1.33 \%)$ & \\
\hline Meropenem & $21(3.12 \%)$ & $21(3.12 \%)$ & \\
\hline Netilmicin & $37(5.50 \%)$ & $4(0.59 \%)$ & $1(0.29 \%)$ \\
\hline Gatifloxacin & $34(5.05 \%)$ & $8(1.19 \%)$ & \\
\hline Cloxacillin & $5(0.74 \%)$ & $37(5.50 \%)$ & \\
\hline Sparfloxacin & $24(3.57 \%)$ & $18(2.67 \%)$ & \\
\hline Total $=672$ & $381(56.69 \%)$ & $284(42.26 \%)$ & $7(1.04 \%)$ \\
\hline
\end{tabular}

Table 2: Isolated drug Methicillin resistance $S$. aureus sensitivity pattern blood sample. Identify drug Methicillin resistance $S$. aureus sensitivity pattern 42 (4.35\%) blood sample. 


\section{Prevalence of Methicillin-Resistant Staphylococcus Aureus of Age and Gender}

Research show that, A total of 964 patients' (outdoor and indoor) blood sample were raised of which $18(1.86 \%)$ were males and $24(2.48 \%)$ were female. The age range of the study members was from $<10$ year to $>80$ year. In a more recent time of the study participants Methicillin Resistant Staphylococcus Aureus (MRSA) most were between the age groups of 31-60. After that, lowest percentage participant's methicillin resistant Staphylococcus aureus was between the age group of 10-30 and 71-90 (Fig. 1).

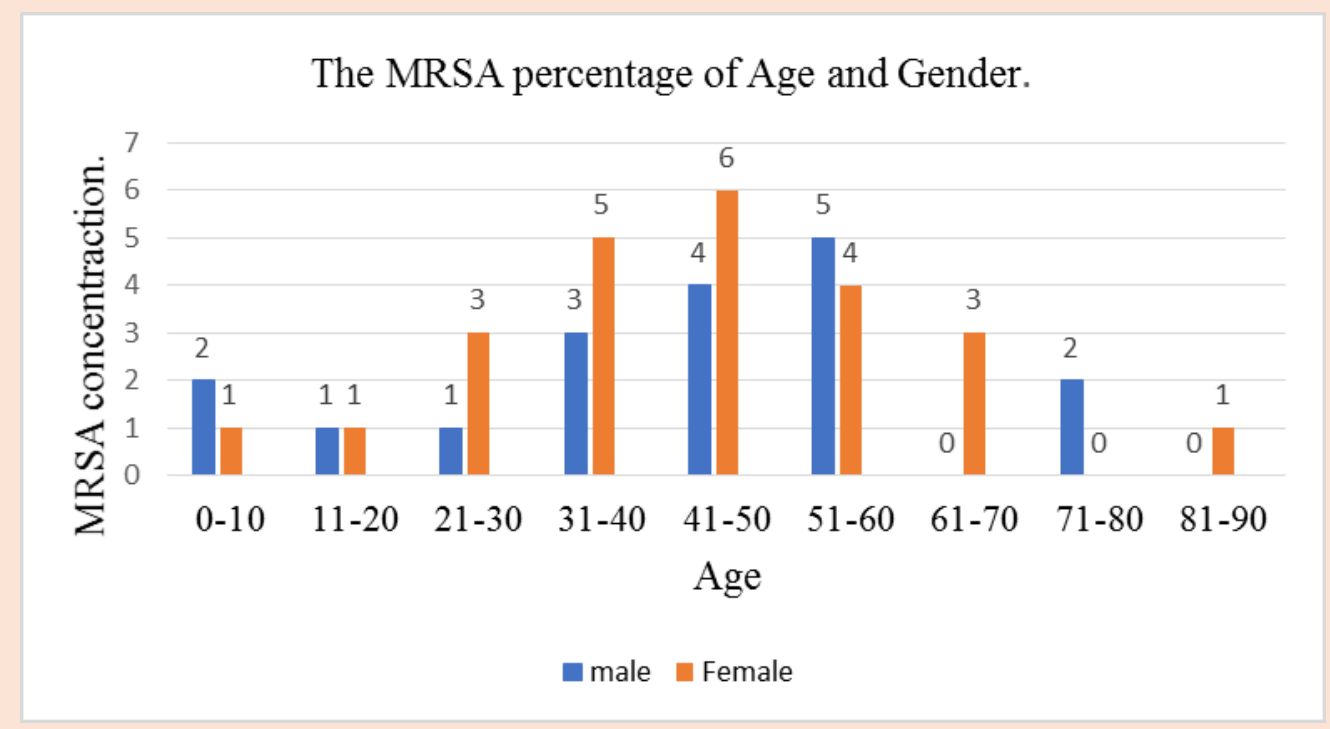

Figure 1: The MRSA percentage of Age and Gender.

\section{Total Bacterial Count of Indoor and Outdoor Patients}

Although this study presents Methicillin-Resistant Staphylococcus Aureus (MRSA) nevertheless, we were found different type of microorganisms among the Methicillin-Resistant Staphylococcus Aureus (MRSA) 42 (4.35\%), were produce Staphylococci aureus. 50 (5.18\%), were produce Salmonella spp. 27 (2.80\%), were produce E. coli, 21 (2.17\%), were found Klebsiella spp. 13 (1.34\%), were produce Pseudomonas spp. 14 (1.45\%), as well as we were not identified (no growth) 797 (82.67\%) (Fig. 2 and Table 3) [17-19]. 


\begin{abstract}
E. coli,
- Salmonella spp.

Klebsiella spp.

Staphylococci aureus.

- Pseudomonas

Methicillin-Resistant

Staphylococcus aureus (MRSA)

No MRSA and other organisms are found (no growth)
\end{abstract}

797

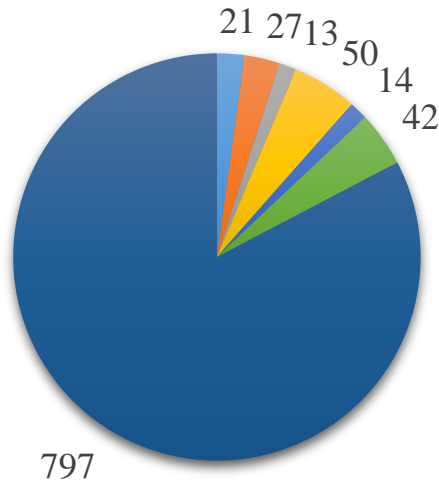

Figure 2: Total count blood sample.

\begin{tabular}{|l|l|l|l|}
\hline Identify Organisms in Total Blood Sample & In-door Patients & Out-door Patients & Total Count \\
\hline E. coli & $15(1.55 \%)$ & $6(0.62 \%)$ & $21(2.17 \%)$ \\
\hline Salmonella spp. & $10(1.03 \%)$ & $17(1.76 \%)$ & $27(2.80 \%)$ \\
\hline Klebsiella spp. & $8(0.82 \%)$ & $5(0.51 \%)$ & $13(1.34 \%)$ \\
\hline Staphylococci aureus. (MSSA) & $40(4.14 \%)$ & $10(1.03 \%)$ & $50(5.18 \%)$ \\
\hline Pseudomonas spp. & $8(0.82 \%)$ & $6(0.62 \%)$ & $14(1.45 \%)$ \\
\hline $\begin{array}{l}\text { Methicillin-Resistant Staphylococcus Aureus } \\
\text { (MRSA) }\end{array}$ & $38(3.94 \%)$ & $4(0.41 \%)$ & $42(4.35 \%)$ \\
\hline $\begin{array}{l}\text { No MRSA, MSSA and other organisms are } \\
\text { found (no growth) }\end{array}$ & $491(50.93 \%)$ & $306(31.74 \%)$ & $797(82.67 \%)$ \\
\hline Total & $\mathbf{6 1 0 ( 6 3 . 2 7 \% )}$ & $\mathbf{3 5 4 ( 3 6 . 7 2 \% )}$ & $\mathbf{9 6 4}(\mathbf{1 0 0 \%})$ \\
\hline
\end{tabular}

Table 3: Total bacterial count of indoor and outdoor patients. 


\section{Conclusion}

Methicillin resistant Staphylococcus aureus has been a cause of human disease comprehensively recorded history. Methicillin Resistant Staphylococcus Aureus (MRSA) infections constitute a vital and still developing public health challenge for globally. This current circumstance applies to MRSA. In this study, we presented data on the frequency of MRSA in Dr. Sirajul Islam Medical Collage and Hospitals Ltd in Dhaka, the capital city of Bangladesh. As a matter of fact, we were identifying most of the affected (MRSA) indoor patients while low affected outdoor patients (Table 1). The common perception is, the greater portion invaded (MRSA) female $(2.48 \%$ ) patients on the other hand, few portions invaded male $(1.86 \%)$ patients (Table 3). The antibiotic period was perhaps extremely prospective to eliminate $S$. aureus (MRSA) as a leading cause of human infections. (Table 2) However, $S$. aureus has remarkable efficiency to develop resistance to antibiotics, which have been the impulse for waves of antibiotic resistance over the past 50 years. We were found MethicillinResistant Staphylococcus Aureus (MRSA) 42 (4.35\%) and other organisms E. coli 21 (2.17\%), Salmonella spp. 27 (2.80\%), Klebsiella spp. 13 (1.34\%), Pseudomonas spp. 14 (1.45\%), Staphylococcus Aureus (MSSA) 50 (5.18\%), No MRSA, MSSA and other organisms are found (no growth) $797(82.67 \%)$ (Table 3). The public health department may have information on other prevalence within the same community or new information on resistance or treatment patterns. These efforts should begin with an agreement upon definitions for outdoor and indoor patients and continue with the improvement of record-based guidance and the implementation of preventive measures to result in better prevention and control of MRSA in our country.

\section{Acknowledgments}

Dr. Sirajul Islam Medical College and Hospital, Department of Microbiology.

\section{References}

1. Lima WW, Peng W, Helen SB, Jessica YW, Kaiwen N, Setoa WH, et al. Determinants of Methicillin-Resistant Staphylococcus Aureus (MRSA) prevalence in the Asia-Pacific region: A systematic review and meta-analysis. J Global Antimicrobial Resist. 2019;14:2213-65.

2. Shamweel A, Mehr-un-Nisa MM, Hiba S. Methicillin Resistant Staphylococcus Aureus (MRSA) a challenge for health care- professionals and patients. Acta Sci. 2017.

3. Goetghebeur M, Landry PA, Han D, Vicente C. Methicillin-resistant Staphylococcus aureus: a public health issue with economic consequences. CN J Infect Dis Medl Microbiol. 2007;18. 
4. Gulani IA, Geidam YA, Adamu L, Lawal JR, Abadam FA. Prevalence and phenotypic detection of methicillin resistance Staphylococcus aureus between ruminants butchered for humanoid intake and animal handlers in Maiduguri, Nigeria. J Adv Veterinary Animal Res. 2016;3(2):152-9.

5. Garoy EY, Gebreab YB, Achila OO, Tekeste DG, Kesete R, Ghirmay R, et al. Methicillin-resistant Staphylococcus aureus (MRSA): prevalence and antimicrobial sensitivity pattern among patients-a multicenter study in Asmara, Eritrea. CN J Infect Dis Medl Microbiol. 2019.

6. Islam MA, Parveen S, Rahman M, Huq M, Nabi A, Khan ZU, et al. Occurrence and characterization of methicillin resistant Staphylococcus aureus in processed raw foods and ready-to-eat foods in an urban setting of a developing country. Frontiers in Microbiol. 2019;10:503.

7. Souza SG, Campos GB, Oliveira PS, Sousa DS, Da Silva DC, Santos VM, Amorim AT, Santos AM, Timenetsky J, Cruz MP, Yatsuda R. Virulence factors in Methicillin-Resistant Staphylococcus aureus isolated from ICU Units in Brazil. Adv Microbiol. 2014.

8. Gallagher R, Motohashi N, Vanam A. Global Methicillin-Resistant Staphylococcus Aureus (MRSA) infections and current research trends. Arch Gen Intern Med. 2017;1(2):3-11.

9. Köck R, Becker K, Cookson B, van Gemert-Pijnen JE, Harbarth S, Kluytmans JA, et al. Methicillin-Resistant Staphylococcus Aureus (MRSA): burden of disease and control challenges in Europe. Eurosurveillance. 2010;15(41):19688.

10. Redziniak DE, Diduch DR, Turman K, Hart J, Grindstaff TL, MacKnight JM, et al. Methicillin-Resistant Staphylococcus Aureus (MRSA) in the Athlete. Int J Sports Med. 2009;30(8):557-62.

11. Chen CJ, Huang YC. New epidemiology of Staphylococcus aureus infection in Asia. Clin Microbiol Infect. 2014;20(7):605-23.

12. Wong JW, Ip M, Tang A, Wei VW, Wong SY, Riley S, et al. Prevalence and risk factors of communityassociated methicillin-resistant Staphylococcus aureus carriage in Asia-Pacific region from 2000 to 2016: a systematic review and meta-analysis. Clin Epidemiol. 2018;10:1489.

13. Phokhaphan P, Tingpej P, Apisarnthanarak A, Kondo S. Prevalence and antibiotic susceptiblity of methicillin resistant staphylococcus aureus, collected at Thammasat University Hospital, Thailand, August 2012-July 2015. Southeast Asian J Trop Med Public Health. 2017;48(2):351-9.

14. Shrestha B. Comparative prevalence of MRSA in two Nepalese tertiary care hospitals. Open J Clin Diagnost. 2013;3:67-73.

15. Papadopoulos P, Papadopoulos T, Angelidis AS, Boukouvala E, Zdragas A, Papa A, et al. Prevalence of Staphylococcus aureus and of Methicillin-Resistant S. Aureus (MRSA) along the production chain of dairy products in north-western Greece. Food Microbiol. 2018;69:43-50.

16. Wayne PA. Clinical and Laboratory Standards Institute, CLSI,(2009). Performance standards for antimicrobial disk susceptibility tests. 2010 .

17. DeLeo FR, Otto M, Kreiswirth BN, Chambers HF. Community-associated meticillin-resistant Staphylococcus aureus. The Lancet. 2010;375(9725):1557-68.

18. Ray P, Gautam V, Singh R. Methicillin-Resistant Staphylococcus Aureus (MRSA) in developing and developed countries: implications and solutions. Regional Health Forum. 2011:15:1-8.

19. Souza SG, Campos GB, Oliveira PS, Sousa DS, Da Silva DC, Santos VM, et al. Virulence factors in Methicillin-Resistant Staphylococcus aureus isolated from ICU Units in Brazil. Adv Microbiol. 2014.

Rahman AM | Volume 1; Issue 2 (2020) | JCIM-1(2)-010 | Research Article

Citation: Rahman AM. Methicillin-Resistant Staphylococcus Aureus (MRSA): Prevalence and Antimicrobial Sensitivity Pattern among Outdoor and Indoor Patients Dhaka City Hospital, Bangladesh. J Clin Immunol Microbiol. 2020;1(2):1-9. 